\title{
ELECTRON BACKSCATTER DIFFRACTION CRYSTAL MORPHOLOGY ANALYSIS AND MULTISCALE SIMULATION OF PIEZOELECTRIC MATERIALS
}

\author{
Y. UETSUJI ${ }^{1}$, H. KURAMAE 2 , K. TSUCHIYA ${ }^{3} \&$ M. KAMLAH ${ }^{4}$ \\ ${ }^{1}$ Department of Mechanical Engineering, Osaka Institute of Technology, Japan. \\ ${ }^{2}$ Department of Technology Management, Osaka Institute of Technology, Japan. \\ ${ }^{3}$ Department of Precision Engineering, Tokai University, Japan. \\ ${ }^{4}$ Institute for Applied Materials, Karlsruhe Institute of Technology, Germany.
}

\begin{abstract}
A computational approach based on electron backscatter diffraction (EBSD) measurement was proposed to estimate the effects of crystal morphology on the overall response of polycrystalline piezoelectric ceramics. EBSD-measured crystal orientations of a polycrystalline piezoelectric ceramic, barium titanate, were applied to a multiscale finite element simulation based on asymptotic homogenization theory. First, the orientation dependence of material properties, such as elastic compliance constants, dielectric and piezoelectric strain constants, was discussed for a single-domain crystal of tetragonal perovskite structure. The computation indicated that piezoelectric strain constants are more sensitive to crystal orientation compared with other properties. Then the single-crystalline material properties were introduced into multidirectionally oriented grains in the polycrystalline microstructure, the multiscale finite element analysis between macrostructure and EBSD-measured microstructure was performed. In this paper emphasis was placed on the diminution of microstructure. The authors discussed about the adverse effect on each component of macrostructural homogenized material properties, which is useful for micromechanics approaches.
\end{abstract}

Keywords: EBSD, homogenization, multiscale simulation, piezoelectric material, polycrystalline.

\section{INTRODUCTION}

Applications of piezoelectric and ferroelectric materials have increased in recent years and they are fueled by developing advanced mechanical electro devices and information technology devises, such as MEMS, $\mu$-TAS, FeRAM, and so on. The important features of these materials are produced from noncentrosymmetric crystal structure, which are typified by tetragonal and rhombohedral perovskite structures. Domain and grain consisting of the asymmetric perovskite structures, which have spontaneous strain and polarization at specific directions, show strong anisotropy in mechanical and electrical behaviors. Therefore, it is important for the material design, processing, and applications, to consider crystal orientation and its distribution.

For various applications, polycrystalline piezoelectric ceramics and thin films are industrially important and more attractive than single crystals. There are obvious differences among polycrystals, thin films, and single crystals in their crystal morphologies. The single crystals have a uniform orientation. The polycrystalline ceramics and the thin films have inhomogeneous structure of multidomain and multigrain aggregates, and they consist of multiple orientations. In general, polycrystalline ceramics include randomly orienting grains and have the transverse isotropy. In case of thin films, one or some specific orientations selectively grow on the substrate. Namely epitaxial growth enables us to fabricate the intended domain and grain configurations and output the remarkable functional performance. At the viewpoint of crystal morphology, polycrystalline ceramics have a large number of orientations 
and more complicated texture than thin films. In any cases, it is clear that texture plays an important role in their effective material properties.

Electron backscatter diffraction (EBSD) measurement $[1,2]$ is generally very useful in analyzing crystal morphology in domain and grain aggregates. In case of piezoelectric and ferroelectric materials, EBSD measurement has been implemented for lead titanate $\left(\mathrm{PbTiO}_{3}\right)$ [3], barium titanate $\left(\mathrm{BaTiO}_{3}\right)$ [4,5], lead zirconium titanate (PZT) [6,7], lead lanthanum zirconium (PLZT) [8], and lead magnesium niobium titanate (PMNT) [9]. In addition, EBSD measurement has been applied to multiferroic materials such as a composite of $\mathrm{BaTiO}_{3}$ and niobium zirconium ferrite [10]. Many previous investigations succeeded in understanding the complicated domain/grain structures and characterizing domain switching under external loads. However, investigations on the relation of realistic microstructure with effective material properties is still lacking for these functional ceramics. On the other hand, advanced computational approaches, finite element simulations incorporating the EBSD-measured microstructure were proposed for polycrystalline steels $[11,12]$. These approaches succeeded in evaluating material properties and behaviors of actual polycrystalline materials. Naturally, the realistic modeling based on EBSD measurement will have promising result for piezoelectric and ferroelectric materials.

On the other hand, there are a lot of micromechanical approaches related on the piezoelectric and ferroelectric materials to evaluate the effect of texture on effective material properties. The effective material properties of unpoled piezoelectric materials have been calculated through theoretical approaches and mathematical simulations [13-15]. The relation between microstructure and effective material properties was estimated by the self-consistent approach for poled piezoelectric polycrystalline ceramics and thin films [16]. For linear piezoelectric problem, new computational approaches, such as meshless, extended finite element methods $[17,18]$, and scale-bridging method [19] were proposed. Especially, although limited in scale-bridging method, the applications to polycrystalline piezoelectric ceramics were reported [20,21]. For nonlinear static problem including domain switching in ferroelectric materials, various finite element simulations were reported for single crystals [22] and polycrystals [23-25]. Additionally phase field simulations were developed for nonlinear thermodynamic problem of ferroelectric materials [26-28]. Many approaches had some contributions in understanding expression mechanism of material performance through microstructural texture. However, all approaches employed some hypothetical models based on Gaussian distribution and orientation distribution function for microstructural texture. There is no research to utilize a realistic model based on measured orientation distribution for piezoelectric and ferroelectric materials.

In our previous studies, we have analyzed the microstructure of $\mathrm{BaTiO}_{3}[29,30]$ and PZT[31] by mean of EBSD. We focused on polycrystalline piezoelectric ceramics because they have most difficult microstructure for numerical modeling. With the aim of a high resolution crystal orientation map, a conductive layer of amorphous osmium was utilized to remove an electric charge on the specimens. Furthermore, we have developed a multiscale finite element analytical code based on asymptotic homogenization theory [20], which can enable us to estimate the macroscopic homogenized material properties from heterogeneous microstructures and evaluate the microscopic mechanical and electrical behaviors in response to the external load. The developed analytical code was applied to EBSD-measured textures of polycrystalline piezoelectric ceramics. First [30], macroscopic homogenized material properties and microscopic response under an electric field were investigated for a twodimensional distribution of EBSD-measured orientations and the validity of the proposed 
multiscale simulation using EBSD-measured texture was confirmed through the trial computation. Second [32], a three-dimensional microstructure model was constructed by stacking in-plane distributions of EBSD-measured orientations, which were obtained in series along the out-of-plane direction. The comparison of macroscopic homogenized material properties between two-dimensional and three-dimensional models was presented by using large microstructure models. Although a parallel iterative computing [33] enabled us to calculate large microstructure models in the above simulations, the diminution of microstructure models are often constrained because of computation environments and experimental limitations. The previous studies indicated the whole tendency that the error of homogenized material properties increases as the microstructure becomes small. However, no attempt has been made to estimate the adverse effect on each component of macroscopic homogenized material properties by diminution of microstructure model.

In this work, we will estimate the individual errors of each component of elastic compliance constants, dielectric constants, and piezoelectric strain constants in the case of reduced microstructures. Especially emphasis is placed on increase and decrease of each component compared with the large microstructure model and difference between in-plane symmetric directions. The paper is organized in the following manner. Basic equations of multiscale simulations based on homogenization theory are introduced in Section 2. The outline of EBSD measurement and the distribution of crystal orientations obtained from a polycrystalline $\mathrm{BaTiO}_{3}$ are explained in Section 3. At the beginning of Section 4, the orientation dependence of single-crystalline material properties, which are inputted into each grain in microstructure, is discussed to consider the influence on macroscopic homogenized material properties. The validity of homogenized material properties obtained from the largest microstructure model is then shown by the comparison with the experimental results. Finally, the computational results and discussion on homogenized material properties of three different-sized microstructures are presented.

\section{COMPUTATIONAL TECHNIQUES}

A three-dimensional multiscale finite element simulation based on homogenization theory was employed to investigate the relationship between the macrostructure and the EBSDmeasured polycrystalline microstructure. The details of formulations and computational approach were presented in the published literatures [20]. The outline of multiscale modeling is introduced in this section.

Figure 1 illustrates the scheme of multiscale modeling for polycrystalline piezoelectric materials. A macrostructure is large compared with microstructures and it can be assumed that microstructures have the periodicity at a microscopic scale. In that case, the multiscale simulation based on homogenization theory enables us to estimate the macrostructural homogenized material properties from heterogeneous microstructures. And it also enables us to evaluate the microscopic mechanical and electrical behaviors in response to the external load applied to the macrostructure. $\boldsymbol{x}=\left(x_{1}, x_{2}, x_{3}\right)^{\mathrm{T}}$ and $\boldsymbol{y}=\left(y_{1}, y_{2}, y_{3}\right)^{\mathrm{T}}$ are employed for macrostructural and microstructural coordinate systems, respectively. Both coordinates can be related by using scale ratio $(\lambda)$ as shown in eqn (1).

$$
y=\frac{x}{\lambda}
$$

Consider an overall structure $\left(\Omega^{\lambda}=\Omega \times Y\right)$ of interest, which consists of macrostructure $(\Omega)$ and microstructure $(Y)$. Displacement $u_{i}^{\lambda}$ and electric potential $\left(\phi^{\lambda}\right)$ of overall structure are 


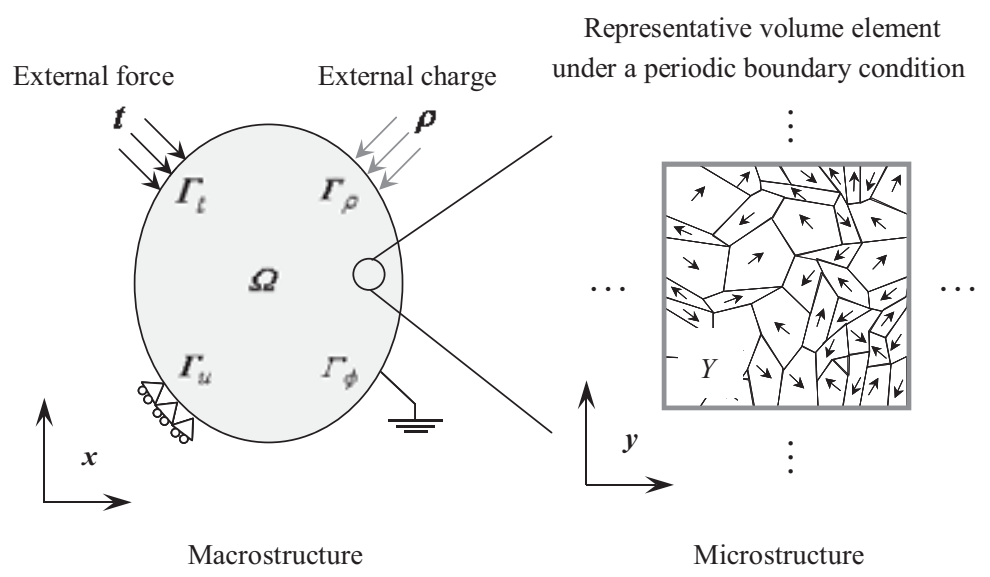

Figure 1: Multiscale modeling of polycrystalline piezoelectric materials. Macrostructure has the same scale with the overall structure of interest and it has homogenized material properties. Microstructure is an aggregate of many grains and it has periodicity at a microscopic scale.

expressed by asymptotic expansion of scale ratio. Only the first-order variation terms are considered in the present two-scale simulation.

$$
\begin{aligned}
& u_{i}^{\lambda}=u_{i}^{0}(\boldsymbol{x})+\lambda u_{i}^{1}(\boldsymbol{x}, \boldsymbol{y})+\cdots \\
& \phi^{\lambda}=\phi^{0}(\boldsymbol{x})+\lambda \phi^{1}(\boldsymbol{x}, \boldsymbol{y})+\cdots
\end{aligned}
$$

where the first terms on the right side $\left(u_{i}^{0}(x)\right.$ and $\left.\phi^{0}(\boldsymbol{x})\right)$ mean macrostructural displacement and electric potential, respectively. The second terms $\left(u_{i}^{1}(x, y)\right.$ and $\left.f^{1}(\boldsymbol{x}, \boldsymbol{y})\right)$ are microstructural perturbations of displacement and electric potential, which are caused by inhomogeneous feature at a microscopic scale. Now, microstructural perturbations are assumed to be proportionate to macrostructural strain and electric field as shown in eqns (4) and (5).

$$
\begin{aligned}
& u_{i}^{1}(\boldsymbol{x}, \boldsymbol{y})=\chi_{i k l}^{u-\varepsilon}(\boldsymbol{x}, \boldsymbol{y}) \frac{\partial u_{k}^{0}(\boldsymbol{x})}{\partial x_{l}}+\chi_{i m}^{u-E}(\boldsymbol{x}, \boldsymbol{y}) \frac{\partial \phi^{0}(\boldsymbol{x})}{\partial x_{m}} \\
& \varphi^{1}(\boldsymbol{x}, \boldsymbol{y})=\chi_{k l}^{\phi-\varepsilon}(\boldsymbol{x}, \boldsymbol{y}) \frac{\partial u_{k}^{0}(\boldsymbol{x})}{\partial x_{l}}+\chi_{m}^{\phi-E}(\boldsymbol{x}, \boldsymbol{y}) \frac{\partial \phi^{0}(\boldsymbol{x})}{\partial x_{m}}
\end{aligned}
$$

where proportional constants $\left(\chi_{i k l}^{u-\varepsilon}, \chi_{i m}^{u-E}, \chi_{k l}^{\phi-\varepsilon}\right.$ and $\left.\chi_{m}^{\phi-E}\right)$ are characteristic functions of displacement, electric potential, and piezoelectric effect.

The above equations are introduced into the principle of virtual work for the overall structure. As a result, microstructural equations can be obtained as follows:

$$
\int_{Y}\left({ }^{\text {micro }} C_{i j p q}^{E} \frac{\partial \chi_{p k l}^{u-\varepsilon}(\boldsymbol{x}, \boldsymbol{y})}{\partial y_{q}}+{ }^{\text {micro }} e_{r i j} \frac{\partial \chi_{k l}^{\phi-\varepsilon}(\boldsymbol{x}, \boldsymbol{y})}{\partial y_{r}}\right) \frac{\partial \delta u_{i}^{1}(\boldsymbol{x}, \boldsymbol{y})}{\partial y_{j}} \mathrm{~d} Y=-\int_{Y}{ }^{\text {micro }} C_{i j k l}^{E} \frac{\partial \delta u_{i}^{1}(\boldsymbol{x}, \boldsymbol{y})}{\partial y_{j}} \mathrm{~d} Y
$$




$$
\begin{aligned}
& \int_{Y}\left({ }^{\text {micro }} C_{i j p q}^{E} \frac{\partial \chi_{p m}^{u-E}(\boldsymbol{x}, \boldsymbol{y})}{\partial y_{q}}+{ }^{\text {micro }} e_{r i j} \frac{\partial \chi_{m}^{\phi-E}(\boldsymbol{x}, \boldsymbol{y})}{\partial y_{r}}\right) \frac{\partial \delta u_{i}^{1}(\boldsymbol{x}, \boldsymbol{y})}{\partial y_{j}} \mathrm{~d} Y=-\int_{Y}{ }^{\text {micro }} e_{m i j} \frac{\partial \delta u_{i}^{1}(\boldsymbol{x}, \boldsymbol{y})}{\partial y_{j}} \mathrm{~d} Y \\
& \int_{Y}\left({ }^{{ }^{\text {micro }}} e_{i p q} \frac{\partial \chi_{p k l}^{u-\varepsilon}(\boldsymbol{x}, \boldsymbol{y})}{\partial y_{q}}-{ }^{\text {micro }} \in_{i r}^{S} \frac{\partial \chi_{k l}^{\phi-\varepsilon}(\boldsymbol{x}, \boldsymbol{y})}{\partial y_{r}}\right) \frac{\partial \delta \phi^{1}(\boldsymbol{x}, \boldsymbol{y})}{\partial y_{i}} \mathrm{~d} Y=-\int_{Y}{ }^{\text {micro }} e_{i k l} \frac{\partial \delta \phi^{1}(\boldsymbol{x}, \boldsymbol{y})}{\partial y_{i}} \mathrm{~d} Y \\
& \int_{Y}\left({ }^{\text {micro }} e_{i p q} \frac{\partial \chi_{p m}^{u-E}(\boldsymbol{x}, \boldsymbol{y})}{\partial y_{q}}-{ }^{\text {micro }} \in_{i r}^{S} \frac{\partial \chi_{m}^{\phi-E}(\boldsymbol{x}, \boldsymbol{y})}{\partial y_{r}}\right) \frac{\partial \delta \phi^{1}(\boldsymbol{x}, \boldsymbol{y})}{\partial y_{i}} \mathrm{~d} Y=\int_{Y}{ }^{\text {micro }} \in_{i m}^{S} \frac{\partial \delta \phi^{1}(\boldsymbol{x}, \boldsymbol{y})}{\partial y_{i}} \mathrm{~d} Y
\end{aligned}
$$

where ${ }^{\text {micro }} C_{i j k l}{ }^{E}$, ${ }^{\text {micro }} \in{ }_{i m}{ }^{S}$, and ${ }^{\text {micro }} e_{m i j}$ denote elastic stiffness constants, dielectric constants, and piezoelectric stress constants of the microstructure, respectively. If all of microstructural equations are solved under a periodic boundary condition, we can obtain characteristic functions of the microstructure. On the other hand, macrostructural equations can be written as follows:

$$
\begin{aligned}
& \int_{\Omega}\left({ }^{\text {macro }} C_{i j k l}^{E} \frac{\partial u_{k}^{0}(\boldsymbol{x})}{\partial x_{l}}+{ }^{\text {macro }} e_{m i j} \frac{\partial \phi^{0}(\boldsymbol{x})}{\partial x_{m}}\right) \frac{\partial \delta u_{i}^{0}(\boldsymbol{x})}{\partial x_{j}} \mathrm{~d} \Omega=\int_{\Gamma_{d}} t_{i} \delta u_{i}^{0}(\boldsymbol{x}) \mathrm{d} \Gamma \\
& \int_{\Omega}\left({ }^{\text {macro }} e_{i k l} \frac{\partial u_{k}^{0}(\boldsymbol{x})}{\partial x_{l}}-{ }^{\text {macro }} \in_{i m}^{S} \frac{\partial \phi^{0}(\boldsymbol{x})}{\partial x_{m}}\right) \frac{\partial \delta \phi^{0}(\boldsymbol{x})}{\partial x_{i}} \mathrm{~d} \Omega=\int_{\Gamma_{e}} \rho \delta \phi^{0}(\boldsymbol{x}) \mathrm{d} \Gamma
\end{aligned}
$$

Macrostructural equations yield to the same formulations with a conventional single-scale problem, where parameters with upper index 'macro' mean homogenized material properties of the macrostructure. They can be obtained from the characteristic functions of the microstructure, as given by

$$
\begin{aligned}
{ }^{\text {macro } C_{i j k l}^{E}}= & \frac{1}{|Y|} \int_{Y}\left({ }^{\text {micro }} C_{i j k l}^{E}+{ }^{\text {micro }} C_{i j p q}^{E} \frac{\partial \chi_{p k l}^{u-\varepsilon}(\boldsymbol{x}, \boldsymbol{y})}{\partial y_{q}}+{ }^{\text {micro }} e_{r i j} \frac{\partial \chi_{k l}^{\varphi-\varepsilon}(\boldsymbol{x}, \boldsymbol{y})}{\partial y_{r}}\right) \mathrm{d} Y \\
{ }^{\text {macro }} e_{m i j} & =\frac{1}{|Y|} \int_{Y}\left({ }^{\text {micro }} e_{m i j}+{ }^{\text {micro }} e_{r i j} \frac{\partial \chi_{m}^{\phi-E}(\boldsymbol{x}, \boldsymbol{y})}{\partial y_{r}}+{ }^{\text {micro }} C_{i j p q}^{E} \frac{\partial \chi_{p m}^{u-E}(\boldsymbol{x}, \boldsymbol{y})}{\partial y_{q}}\right) \mathrm{d} Y \\
& =\frac{1}{|Y|} \int_{Y}\left({ }^{\text {micro }} e_{m i j}+{ }^{\text {micro }} e_{m p q} \frac{\partial \chi_{p i j}^{u-\varepsilon}(\boldsymbol{x}, \boldsymbol{y})}{\partial y_{q}}-{ }^{\text {micro }} \in_{m r}^{S} \frac{\partial \chi_{i j}^{\phi-\varepsilon}(\boldsymbol{x}, \boldsymbol{y})}{\partial y_{r}}\right) \mathrm{d} Y \\
{ }^{\text {macro }} \in_{i m}^{S} & =\frac{1}{|Y|} \int_{Y}\left({ }^{\text {micro }} \in_{i m}^{S}+{ }^{\text {micro }} \in \in_{i r}^{S} \frac{\partial \chi_{m}^{\phi-E}(\boldsymbol{x}, \boldsymbol{y})}{\partial y_{r}}-{ }^{\text {micro }} e_{i p q} \frac{\partial \chi_{p m}^{u-E}(\boldsymbol{x}, \boldsymbol{y})}{\partial y_{q}}\right) \mathrm{d} Y
\end{aligned}
$$

An isoparametric solid element with eight nodes at the corners is employed for finite element modeling of microstructures and macrostructures. A two-scale finite element analytical code was developed to solve the above microstructural and macrostructural correlative equations and is used here to evaluate the macrostructural homogenized properties for EBSD-measured microstructure of polycrystalline piezoelectric ceramics. 


\section{EBSD CRYSTAL MORPHOLOGY ANALYSIS}

$\mathrm{A} \mathrm{BaTiO}_{3}$ polycrystalline piezoelectric ceramic (Murata Manufacturing Co. Ltd) was used for the EBSD specimen. The specimen was a circular disk of $15 \mathrm{~mm}$ diameter and $1 \mathrm{~mm}$ thickness, and it was electrically poled along the thickness direction. The crystal structure was examined by mean of X-ray diffraction (XRD). XRD spectrum indicated that it had a perovskite tetragonal structure and the lattice constants of $a$ and $c$ axes were $0.3995 \mathrm{~nm}$ and $0.4034 \mathrm{~nm}$, respectively.

The observed surface was mechanically polished for 20 min using $3 \mathrm{~mm}$ diamond particles (Marumoto Struers Co., DP-Spray) with a polishing sheet (Marumoto Struers Co., DP-Mol). And then, it was chemically polished at pH 3.5 for 20 min using colloidal particles (Marumoto Struers Co., OP-A) with a polishing sheet (DP-Chem). The surface of the insulative specimen was coated with the electrical conductive and amorphous osmium layer to defend the electrification due to electron beam. We employed an osmium coater (Meiwafosis Co., Ltd, Neoc-ST) and set the discharging time in osmium gas to 10s. The estimated thickness of osmium layer is $0.5 \mathrm{~nm} / \mathrm{s}$. In addition, a silver paste (Fujikura Kasei Co. Ltd, type D-500) was applied to the surface except for the EBSD measurement region to leak the accumulated charge.

Figure 2 shows the crystal morphology of polycrystalline $\mathrm{BaTiO}_{3}$ obtained by means of EBSD (Oxford Instruments PLC, Link ISIS C.7272) implemented in SEM (JEOL Datum Ltd, JSM-5410). The scanning interval was set to $0.64 \mu \mathrm{m}$ and the scanning area was $90.8 \times 90.8 \mu \mathrm{m}^{2}$. The mean diameter of grain was estimated to be $\sim 6.71 \mu \mathrm{m}$. This figure demonstrates (a) the crystal orientation map and (b) (001) pole figure. If estimated from the mean diameter of grain, the sampling area includes $\sim 233$ grains. In the crystal orientation map, the frequency of the [001], [111], and [101] directions is higher than that of the [110] and [100] directions.

\section{MULTISCALE SIMULATION}

\subsection{Orientation dependence of single crystal}

Before we discuss the relationship between the macroscopic homogenized material properties and the polycrystalline microstructure through the multiscale finite element simulation, it is necessary to understand the characteristics of grain as a compositional unit of the

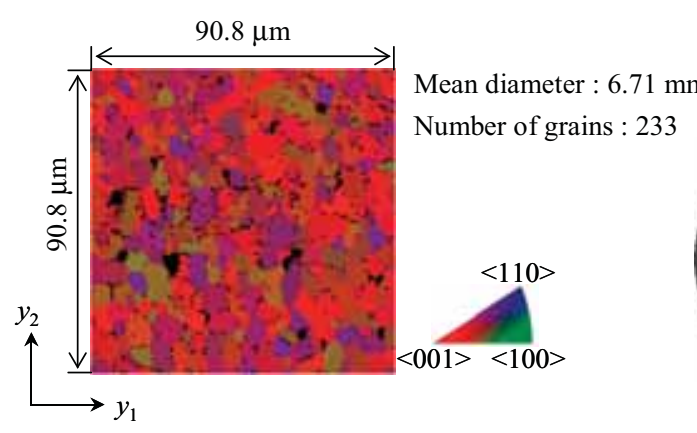

(a) Crystal orientation map

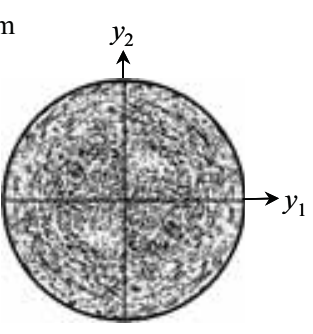

(b) (001) pole figure

Figure 2: Crystal morphology of a polycrystalline $\mathrm{BaTiO}_{3}$ obtained by means of EBSD. The scanning area is $90.8 \times 90.8 \mu \mathrm{m}^{2}$ which consists of $\sim 233$ grains. 
microstructure. In the latter computation, the material properties of single-domain crystalline $\mathrm{BaTiO}_{3}$ [34] were inputted into each grain in the polycrystalline microstructure. Every grain significantly changes the mechanical and electrical behaviors according to its crystal orientation. Consequently, the orientation dependence of material properties was investigated for single crystalline $\mathrm{BaTiO}_{3}$ with perovskite tetragonal structure.

Figure 3 shows the orientation dependence of material properties for single crystalline $\mathrm{BaTiO}_{3}$. The material properties were obtained through coordinate transform during $180^{\circ}$ rotation in a counterclockwise direction around the second axis of crystallographic coordinate system. The horizontal axis means rotation angle, which indicates the angle between the direction of spontaneous polarization and the third axis of global coordinate system. When the rotation angle increases from $0^{\circ}$ to $180^{\circ}$, the crystallographic direction orienting to the third axis of global coordinate system is changed from the [001] direction to the inverse one through the [101] and the [100] directions; (a) elastic compliance constants and (b) dielectric constants indicate the normal tendency of transverse isotropic materials, the extreme values of which appear according to $90^{\circ}$ rotation. On the other hand, (c) piezoelectric strain constants show the complicated dependence on crystal orientation. The components of $d_{333}$ and $d_{311}$ have the maximum peak at the [101] direction. This is the result that normal strains and shear strain caused by piezoelectric effect join together and make total strain larger at a specific off-axis direction.

\subsection{Macroscopic homogenized material properties of polycrystalline microstructure}

Figure 4 shows the finite element modeling of EBSD-measured realistic polycrystalline microstructure. The EBSD-measured crystal orientations within a square region of $90.8 \times$ $90.8 \mu \mathrm{m}^{2}$ were sampled for computations. Plate-shaped regular cubic meshes, whose number of elements corresponds exactly to one of measuring points, were employed for microstructural finite element models. The crystal orientation of each measuring point was independently mapped into one element. Our previous investigation [32] indicated that the dependence on the size of sampling area of crystal orientations falls off and the relative error of macroscopic homogenized material properties becomes $5 \%$ or less if the sampling area is beyond $\sim 60 \times 60 \mu \mathrm{m}^{2}$. Consequently, the crystal orientation map of $90.8 \times 90.8 \mu \mathrm{m}^{2}$ is large enough to become a representative volume element of microstructure.

Figure 5 shows the full components of macroscopic homogenized material properties obtained by the multiscale simulation using the EBSD-measured microstructure. This figure demonstrates (a) elastic compliance constants, (b) relative dielectric constants, and (c) piezoelectric strain constants which include the experimental values described in a brochure of Murata Manufacturing Co. Ltd. Because of averaging grain properties with various crystal orientations in polycrystalline microstructure, most of macroscopic homogenized material properties lead to an intermediate value between the maximum and the minimum of single crystal as shown in Fig. 3. The computational values of piezoelectric strain constants, ${ }^{\text {macro }} d_{311}$ and ${ }^{\text {macro }} d_{333}$, have reliable correspondence to experimental values, although ${ }^{\text {macro }} d_{333}$ has relatively large error. Then, if we focus attention on the transverse isotropy, the percentage variation between the respective components of a pair is also denoted in this figure. As most of percentage variations are smaller than $1 \%$, the respective components of a pair of macroscopic homogenized material properties shows good agreements with respect to one another.

In case the size of sampling area of EBSD-measured orientations was minified, the variation of macroscopic homogenized material properties was investigated. Three different-sized areas, such as $29.8 \times 29.8 \mu \mathrm{m}^{2}, 55.2 \times 55.2 \mu \mathrm{m}^{2}$ and $80.6 \times 80.6 \mu \mathrm{m}^{2}$, were sampled from the central 


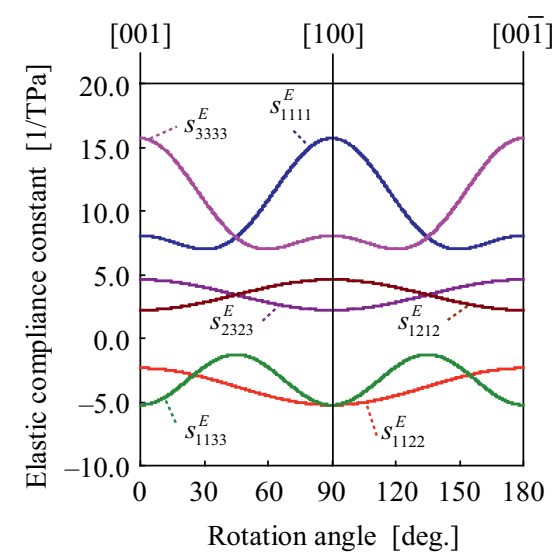

(a) Elastic compliance constants

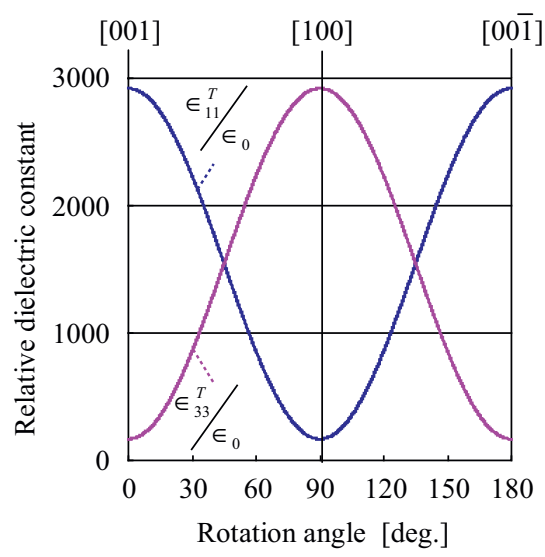

(b) Relative dielectric constants

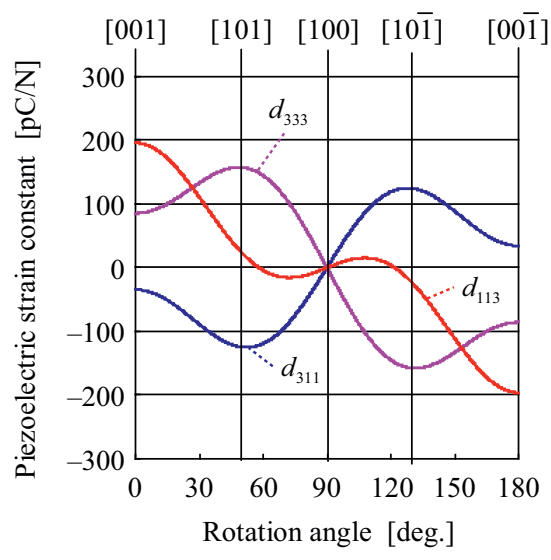

(c) Piezoelectric strain constants

Figure 3: Orientation dependence of material properties for single crystalline $\mathrm{BaTiO}_{3}$. These were obtained through coordinate transform during $180^{\circ}$ rotation in a counterclockwise direction around the second axis of crystallographic coordinate system.

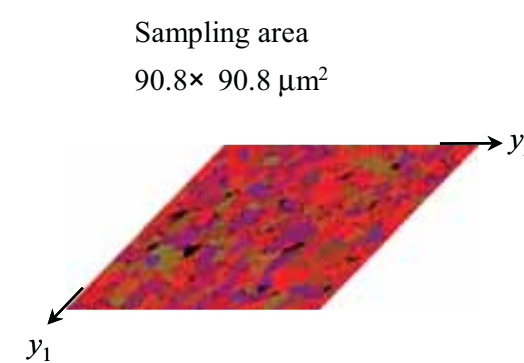

EBSD crystal orientations

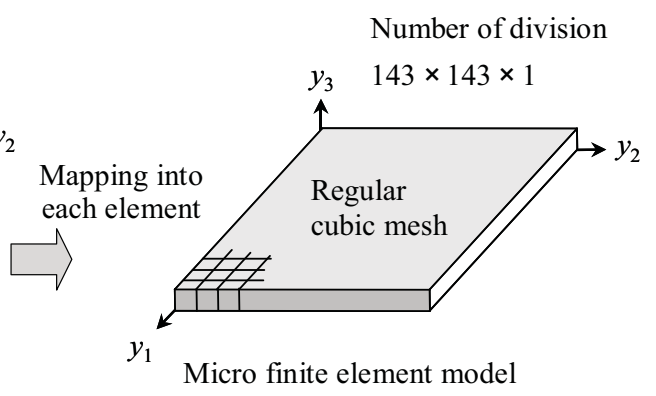

Number of division 


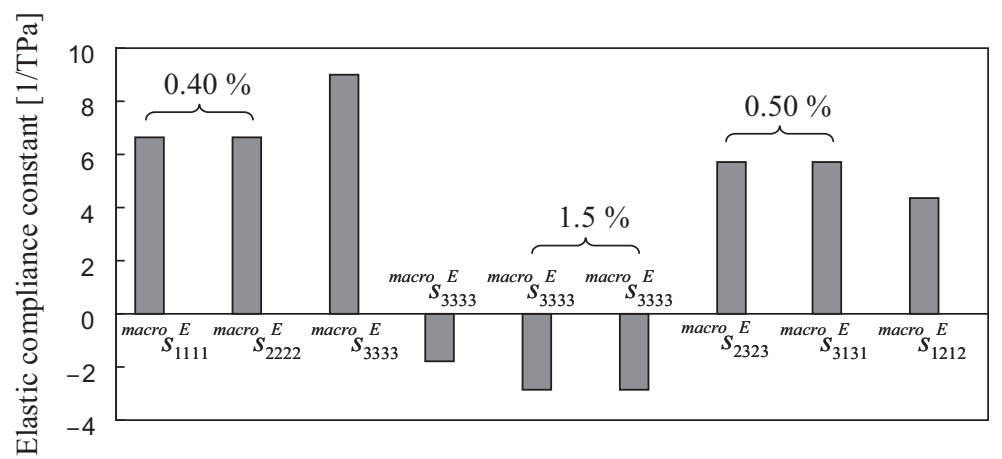

(a) Elastic compliance constants

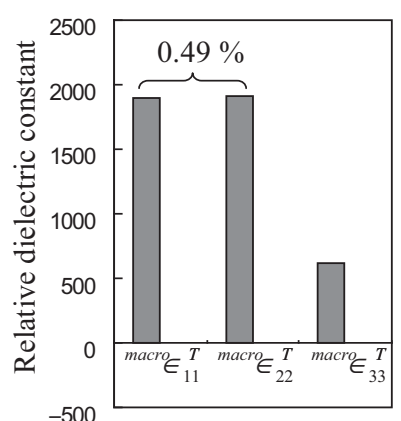

(b) Relative dielectric constants

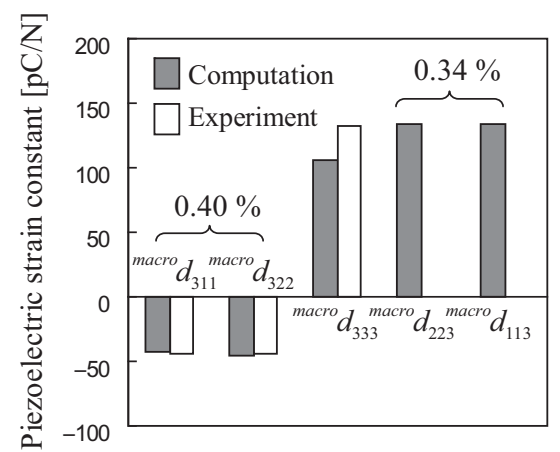

(c) Piezoelectric strain constants

Figure 5: The full components of macroscopic homogenized material properties obtained from the multiscale finite element simulation using the EBSD-measured polycrystalline microstructure. The experimental values of piezoelectric strain constants were provided in a brochure of Murata Manufacturing Co. Ltd.

region of the abovementioned $90.8 \times 90.8 \mu \mathrm{m}^{2}$. These sampling areas include 25,86 , and 184 grains, respectively. The crystal orientations of three sampling areas were individually introduced into regularly divided finite element meshes with correspondence between numbers of elements and measuring points. Then macroscopic homogenized material properties were analyzed for three different-sized microstructures. When computational results were compared to those of the largest microstructure $90.8 \times 90.8 \mu \mathrm{m}^{2}$, Fig. 6 shows the percentage variation of macroscopic homogenized material properties. According to miniaturization of microstructure, the percentage variation of every component increase by graduation. If paying close attention to signs of variation, some components of elastic compliance tensor, $s^{E}{ }_{1111}, s^{E}{ }_{2222}$, $s^{E}{ }_{1122}, s^{E}{ }_{2323}, s^{E}{ }_{3131}, s^{E}{ }_{1212}$, were overvalued; by contrast, the other components were undervalued in case of smaller microstructure. Additionally, in-plane components of dielectric tensor, $\in^{T}{ }_{11}, \in^{T}{ }_{22}$, were undervalued and out-of-plane component, $\in_{33}^{T}$, was overvalued if microstructure is not large enough. On the other hand, there was no distinct tendency in normal components of piezoelectric strain tensor, such as $d_{311}, d_{322}$ and $d_{333}$, although shear components, $d_{223}$ and $d_{113}$, were undervalued in case of smaller microstructure.

In order to discuss the contributing factors of Fig. 6, it is necessary to review the orientation dependence of single crystalline material properties in Fig. 3. We focused on the variation within the range of rotation angle from $0^{\circ}$ to $90^{\circ}$ because microstructure is under approximately 


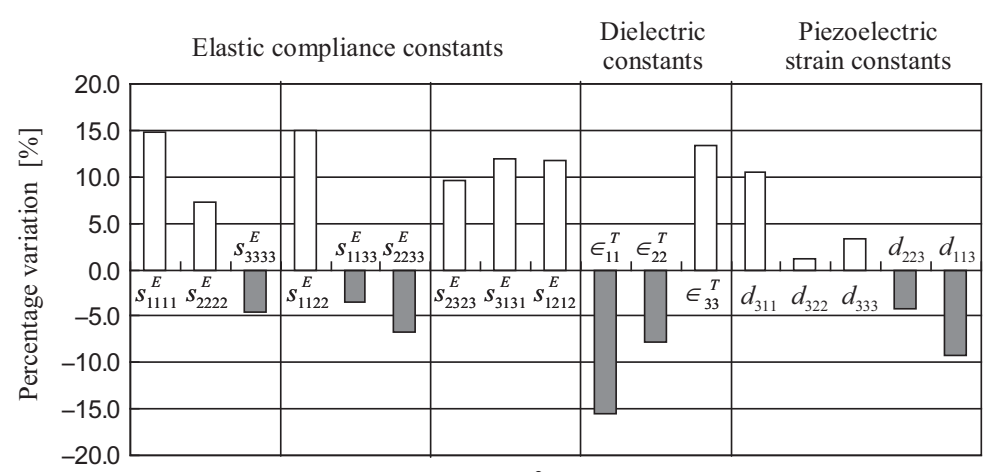

(a) $29.8 \times 29.8 \mu \mathrm{m}^{2}$ including 25 grains

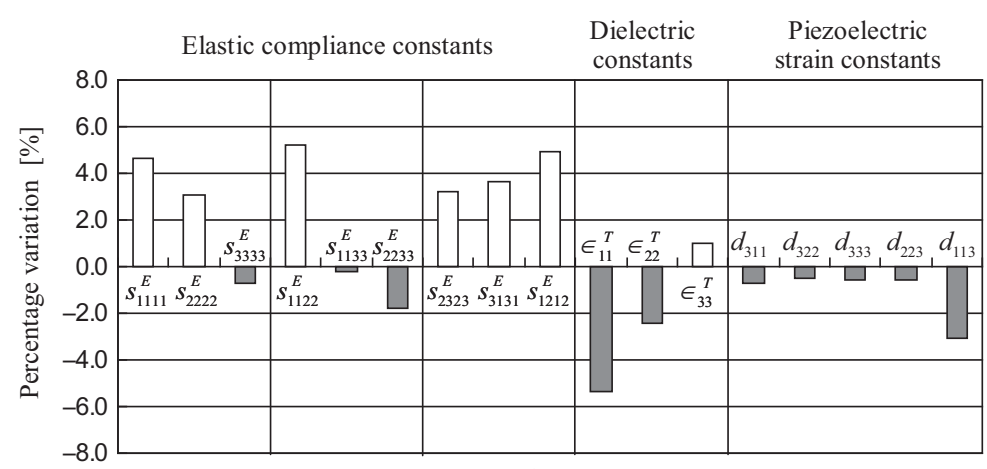

(b) $55.2 \times 55.2 \mu \mathrm{m}^{2}$ including 86 grains

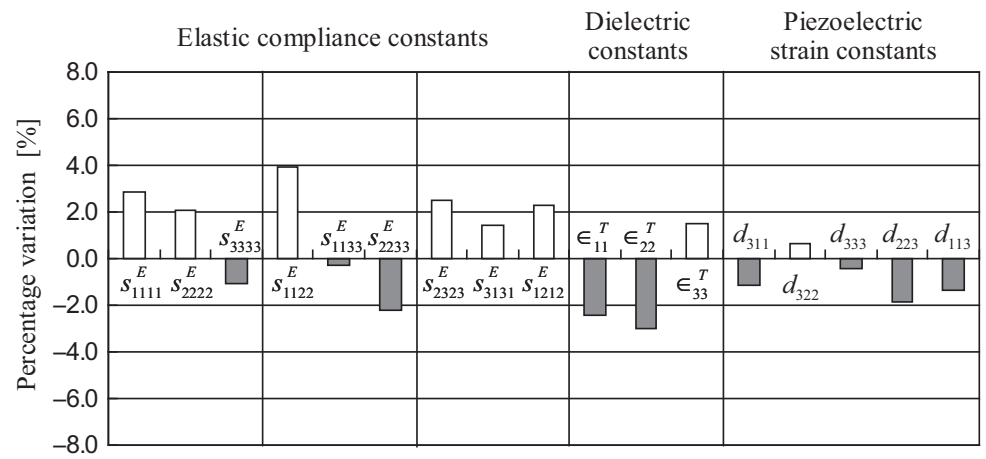

(c) $80.6 \times 80.6 \mu \mathrm{m}^{2}$ including 184 grains

Figure 6: Comparison of the percentage variation of macroscopic homogenized material properties among three different-sized microstructures, which was calculated by difference from those obtained from the largest microstructure of $90.8 \times 90.8 \mu \mathrm{m}^{2}$.

full-poled state. Figure 6 indicates that material properties can be classified into three types: monotone increasing constants $\left(s^{E}{ }_{1111}, s^{E}{ }_{2222}, s^{E}{ }_{1122}, s^{E}{ }_{1212}\right.$, and $\left.\in_{33}^{T}\right)$, monotone decreasing constants $\left(s^{E}{ }_{3333}, s^{E}{ }_{2323}, s^{E}{ }_{3131}, \in^{T}{ }_{11}, \in_{22}^{T}, d_{223}\right.$, and $\left.d_{113}\right)$, and the others $\left(s^{E}{ }_{1133}, s^{E}{ }_{2233}, d_{311}\right.$, $d_{322}$, and $d_{333}$ ). In general, single crystal has strong anisotropy and it shows the maximal and the minimal values of material properties. The monotone increasing and the monotone decreasing constants of polycrystalline material properties become the intermediate values between the maximal and the minimal values of single crystal because they are averaged by 
multi-orientations of grains. If microstructure becomes larger, the number of grains increases and the averaging effect becomes stronger. In contrast, if microstructure is not large enough, monotone decreasing components are overvalued and monotone increasing components are undervalued because of incomplete averaging by multi-orientations. This orientation dependence can compatibly explain computational results of monotone changing components of elastic compliance, dielectric, and piezoelectric strain tensors in Fig. 6. On the other hand, the other components, such as $s^{E}{ }_{1133}, s^{E}{ }_{2233}, d_{311}, d_{322}$, and $d_{333}$, show no steady tendency among different-sizes microstructures because of the complicated orientation dependence.

\section{CONCLUSIONS}

In this paper, we presented a multiscale finite element simulation based on EBSD-measured crystal orientations and reviewed the adverse effect of diminution of microstructure on macroscopic homogenized material properties. As a computational result, the macroscopic homogenized material properties approximately correspond to experimental values and they satisfy the transverse isotropy in case of large microstructure. The specified components of elastic compliance, dielectric, and piezoelectric strain tensors are overvalued or undervalued, according to miniaturization of microstructure. This adverse effect of diminution of microstructure could be explained by the orientation dependence of single crystalline material properties.

\section{ACKNOWLEDGMENTS}

One of the authors (Y. Uetsuji) was financially supported by a Grant-in-Aid for Young Scientists (B) (No. 22760087) from the Ministry of Education, Culture, Sports, Science and Technology of Japan.

\section{REFERENCES}

[1] Venables, J. \& Harland, C., Electron back scattering patterns - A new technique for obtaining crystallographic information in the scanning electron microscope. Philosophical Magazine, 27, pp.1193-1200, 1973. doi: http://dx.doi.org/10.1080/ $\underline{14786437308225827}$

[2] Dingley, D. \& Randel, V., Microstructure determination by electron back-scatter diffraction. Journal of Material Science, 27, pp. 4545-4566, 1992. doi: http://dx.doi. org/10.1007/BF01165988

[3] Yang, L.C., Dumler, I. \& Wayman, C.M., Studies of herringbone domain structures in lead titanate by electron back-scattering patterns. Materials Chemistry and Physics, 36, pp. 282-288, 1994. doi: http://dx.doi.org/10.1016/0254-0584(94)90043-4

[4] Koblischka-Veneva, A. \& Mücklich, F., Orientation imaging microscopy applied to $\mathrm{BaTiO}_{3}$ ceramics. Crystal Engineering, 5, pp. 235-242, 2002. doi: http://dx.doi. org/10.1016/S1463-0184(02)00034-5

[5] Reichmanna, A., Zankela, A., Reingrubera, H., Pölta, P. \& Reichmann, K., Direct observation of ferroelectric domain formation by environmental scanning electron microscopy. Journal of the European Ceramic Society, 31, pp. 2939-2942, 2011. doi: http://dx.doi.org/10.1016/j.jeurceramsoc.2011.05.043

[6] Tai, C.W., Baba-kishi, K.Z. \& Wong, K.H., Microtexture characterization of PZT ceramics and thin films by electron microscopy. Micron, 33, pp. 581-586, 2002. doi: http://dx.doi.org/10.1016/S0968-4328(02)00016-1

[7] Okayasua, M., Satoa, K. \& Kusaba, Y., Domain switching characteristics of lead zirconate titanate piezoelectric ceramics during mechanical compressive loading. Journal of the European Ceramic Society, 31, pp. 129-140, 2011. doi: http://dx.doi. org/10.1016/j.jeurceramsoc.2010.09.001 
[8] Faryna, M., Sztwiertnia, K. \& Sikorski, K., Simultaneous WDXS and EBSD investigations of dense PLZT ceramics. Journal of the European Ceramic Society, 26, pp. 2967-2971, 2006. doi: http://dx.doi.org/10.1016/j.jeurceramsoc.2006.02.013

[9] Samardžija, Z., Jeon, J.H. \& Čeh, M., Microstructural and compositional study of a bulk $\mathrm{Pb}\left(\mathrm{Mg}_{1 / 3} \mathrm{Nb}_{2 / 3}\right) \mathrm{O}_{3}-\mathrm{PbTiO}_{3}$ single crystal grown from a $\mathrm{BaTiO}_{3}$ seed. Materials Characterization, 58, pp. 534-543, 2007. doi: http://dx.doi.org/10.1016/j.matchar.2006.10.003

[10] Koblischka, M.R., Koblischka-Veneva, A., Wicka, M., Mitoseriuc, L. \& Hartmann, U., Microstructure and magnetic properties of $\mathrm{BaTiO}_{3}-(\mathrm{Ni}, \mathrm{Zn}) \mathrm{Fe}_{2} \mathrm{O}_{4}$ multiferroics. Thin Solid Films, 518, pp. 4730-4733, 2010. doi: http://dx.doi.org/10.1016/j.tsf.2009.12.070

[11] Wu, P.D., Lloyd, D.J., Bosland, A., Jin, H. \& MacEwen, S.R., Analysis of roping in AA6111 automotive sheet. Acta Materialia, 51, pp. 1945-1957, 2003. doi: http:// dx.doi.org/10.1016/S1359-6454(02)00600-6

[12] Nakamachi, E., Tam, N.N. \& Morimoto, H., Multi-scale finite element analyses of sheet metals by using SEM-EBSD measured crystallographic RVE models. International Journal of Plasticity, 23, pp. 450-489, 2007. doi: http://dx.doi.org/10.1016/ j.ijplas.2006.06.002

[13] Marutake, M., A calculation of physical constants of ceramics barium titanate, Journal of the Physical Society of Japan, 11, pp. 807-814, 1956. doi: http://dx.doi.org/10.1143/ JPSJ.11.807

[14] Olson, T. \& Avellaneda, M., Effective dielectric and elastic constants of piezoelectric polycrystals. Journal of Applied Physics, 71, pp. 4455-4464, 1992. doi: http://dx.doi. org/10.1063/1.350788

[15] Dunn, M.L., Effects of grain shape anisotropy, porosity, and microcracks on the elastic and dielectric constants of polycrystalline piezoelectric ceramics. Journal of Applied Physics, 78, pp. 1533-1542, 1995. doi: http://dx.doi.org/10.1063/1.360246

[16] Li, J.Y., The effective electroelastic moduli of textured piezoelectric polycrystalline aggregates. Journal of the Mechanics and Physics of Solids, 48, pp. 529-552, 2000. doi: http://dx.doi.org/10.1016/S0022-5096(99)00042-3

[17] Wu, C.P., Chiu, K.H. \& Jiang, R.Y., A meshless collocation method for the coupled analysis of functionally graded piezo-thermo-elastic shells and plates under thermal loads. International Journal of Engineering Science, 56, pp. 29-48, 2012. doi: http:// dx.doi.org/10.1016/j.ijengsci.2012.03.001

[18] Nguyen-Vinh, H., Baker, I., Msekh, M.A., Song, J.H., Muthu, J., Zi, G., Le, P., Bordas, S.P.A., Simpson, R., Natarajan, S., Lahmer, T. \& Rabczuk, T., Extended finite element method for dynamic fracture of piezo-electric materials. Engineering Fracture Mechanics, 92, pp. 19-31, 2012. doi: http://dx.doi.org/10.1016/j.engfracmech.2012.04.025

[19] Nelli Silva, E.C., Ono Fonseca, J.S., Montero F., Crumm, A. T., Brady, G. A., Halloran, J. W. \& Kikuchi, N., Design of piezocomposite materials and piezoelectric transducers using topology optimization. Archives of Computational Methods in Engineering, 6, pp. 117-182, 1999. doi: http://dx.doi.org/10.1007/BF02736183

[20] Uetsuji, Y., Nakamura, Y., Ueda, S. \& Nakamachi, E., Numerical investigation on ferroelectric properties of piezoelectric materials. Modelling and Simulation in Materials Science and Engineering, 12, pp. S303-S317, 2004. doi: http://dx.doi. org/10.1088/0965-0393/12/4/S02

[21] Jayachandran, K.P., Guedes, J.M. \& Rodrigues, H.C., Homogenization of textured as well as randomly oriented ferroelectric polycrystals. Computational Materials Science, 45, pp. 816-820, 2009. doi: http://dx.doi.org/10.1016/j.commatsci.2008.05.027 
[22] Elhadrouz, M., Zineb, T.B. \& Patoor, E., Finite element analysis of a multilayer piezoelectric actuator taking into account the ferroelectric and ferroelastic behaviors. International Journal of Engineering Science, 44, pp. 996-1006, 2006. doi: http:// dx.doi.org/10.1016/j.ijengsci.2006.05.013

[23] Zhang, W., Bhattachary, K., A computational model of ferroelectric domains. Part I: Model formulation and domain switching. Acta Materialia, 53, pp. 185-198, 2005. doi: http://dx.doi.org/10.1016/j.actamat.2004.09.016

[24] Li, F.X. \& Rajapakse, R.K.N.D., Nonlinear finite element modeling of polycrystalline ferroelectrics based on constrained domain switching, Computational Materials Science, 44, pp. 322-329, 2008. doi: http://dx.doi.org/10.1016/j.commatsci.2008.03.040

[25] Li, Q., Ricoeur, A., Enderlein, M. \& Kuna, M., Evaluation of electromechanical coupling effect by microstructural modeling of domain switching in ferroelectrics. Mechanics Research Communications, 37, pp. 332-336, 2010. doi: http://dx.doi.org/10.1016/ j.mechrescom.2010.03.003

[26] Xu, B.X., Schrade, D., Mueller, R. \& Gross, D., Micromechanical analysis of ferroelectric structures by a phase field method. Computational Materials Science, $\mathbf{4 5}$, pp. 832-836, 2009. doi: http://dx.doi.org/10.1016/j.commatsci.2008.07.010

[27] Qiao, H., Wang, J. \& Chen, W., Phase field simulation of domain switching in ferroelectric single crystal with electrically permeable and impermeable cracks. Acta Mechanica Solida Sinica, 25, pp. 1-8, 2012.

[28] Li, F.X. \& Zhou, X.L., Simulations of gradual domain-switching in polycrystalline ferroelectrics using an optimization-based, multidomain-grain model. Computers \& Structures, 89, pp. 1142-1147, 2011. doi: http://dx.doi.org/10.1016/j.compstruc.2010. $\underline{11.002}$

[29] Uetsuji, Y., Yoshida, T., Yamakawa, T., Tsuchiya, K., Ueda, S. \& Nakamachi, E., Evaluation of ferroelectric properties of piezoelectric ceramics based on crystallographic homogenization method and crystal orientation analysis by SEM.EBSD technique. JSME International Journal Series A, 49, pp. 209-215, 2006. doi: http://dx.doi. org/10.1299/jsmea.49.209

[30] Uetsuji, Y., Kuramae, H., Tsuchiya, K. \& Kamlah, M., A multiscale finite element simulation of piezoelectric materials using realistic crystal morphology. WIT Transactions on Modelling and Simulation, 51, pp. 601-611, 2011. doi: http://dx.doi. org/10.2495/CMEM110531

[31] Uetsuji, Y., Satou, Y., Nagakura, H., Nishioka, H., Kukamae, H. \& Tsuchiya, K., Crystal morphology analysis of piezoelectric ceramics using electron backscatter diffraction method and its application to multiscale finite element analysis. Journal of Computational Science and Technology, 2, pp. 568-577, 2008. doi: http://dx.doi. org/10.1299/jest.2.568

[32] Uetsuji, Y., Kimura, S., Kuramae, H., Tsuchiya, K. \& Kamlah, M., Multiscale finite element simulations of piezoelectric materials based on two- and three-dimensional electron backscatter diffraction-measured microstructures. Journal of Intelligent Material Systems and Structures, 23, pp. 563-573, 2012. doi: http://dx.doi.org/ $\underline{10.1177 / 1045389 X 12436740}$

[33] Kuramae, H., Nishioka, H., Uetsuji, Y. \& Nakamachi, E., Development and performance evaluation of parallel iterative method. Transactions of the Japan Society for Computational Engineering and Science, Paper No. 20070033, 2007.

[34] Jaffe, B., Piezoelectric ceramics, Academic press: London and New York, p. 74, 1971. 